\title{
La significación social del patrimonio: Análisis sobre la percepción del patrimonio en la Comunidad de Madrid ${ }^{1}$
}

\author{
Olaia Fontal Merillas, Marta Martínez Rodríguez y Jesús Cepeda Ortega
}

Universidad de Valladolid, España

\section{RESUMEN}

La educación patrimonial se ha consolidado en nuestro país como una disciplina científica en las últimas décadas, constatando la preocupación en materia de patrimonio y educación por parte del Estado con la implantación del Plan Nacional de Educación y Patrimonio. La Comunidad de Madrid ha recogido el testigo y está desarrollando el Plan de Educación Patrimonial, diseñando acciones y productos educativos que se ajusten a las necesidades sociales. Con el objetivo de conocer el punto de partida sobre el que desarrollar este plan, se lleva a cabo la presente investigación que busca analizar las percepciones sociales sobre el patrimonio de la Comunidad de Madrid. Para ello se diseña una entrevista semiestructurada con variables sociodemográficas y variables relativas a la percepción como la tipología patrimonial, la motivación o la accesibilidad al patrimonio. Son entrevistados un total de 122 sujetos en el marco de la XI Bienal Ibérica de Patrimonio Cultural (AR\&PA). Los resultados obtenidos reflejan que las personas tienen una gran discrepancia en relación a la accesibilidad al patrimonio y poseen una percepción tangible del patrimonio relegando a un segundo plano el patrimonio inmaterial. Estos datos suponen una hoja de ruta sobra la cual los organismos encargados de la educación patrimonial deben diseñar acciones más específicas, concretas, sistematizadas y adecuadas a las necesidades sociales de su población.

Palabras clave: Educación patrimonial; Identidad; Plan; Percepción Social; Sensibilización.

\section{Social signification of heritage: An analysis of heritage perception in the Community of Madrid}

\section{ABSTRACT}

Heritage education has been established in our country as a scientific discipline in the last decades, confirming the concern in heritage and education matters by the State with the implementation of the Educational and Heritage National Plan. The Community of Madrid took the helm and is developing the Heritage Education Plan, designing actions and educational products adjusted to social needs. To know the starting point on which this plan may be developed, the present study is carried out to analyse the social perceptions about the heritage of the Community of Madrid. To achieve this, a semi-structured interview is designed including sociodemographic variables and variables related to perception such as heritage typology, motivation or accessibility to heritage. A sample of 122 individuals are interviewed within the XI Bienal Ibérica de Patrimonio Cultural (AR\&PA) framework. Results obtained show that individuals greatly disagree with accessibility to heritage and own a tangible perception of heritage, being immaterial heritage secondary. This results imply a road map on which organizations responsible for heritage education should design more specific, precise and systematized actions that are suitable to the social needs of their population.

Keywords: Heritage Education; Identity; Plan; Social Perception; Awareness.

ISSN: 0210-2773

DOI: https://doi.org/10.17811/rifie.49.1.2020.17-24

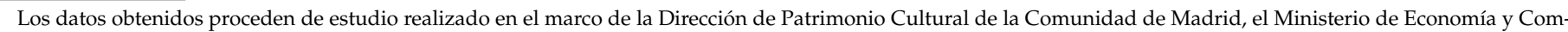

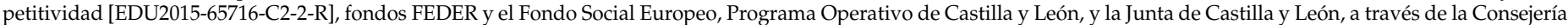
de Educación.
} 


\section{Introducción}

A nivel internacional son muchos los organismos encargados de la tutela y gestión de los bienes patrimoniales que vienen defendiendo y reconociendo a través de tratados y convenciones la necesidad de que la persona y los valores humanos ocupen un lugar central dentro de un concepto más amplio e interdisciplinar de patrimonio cultural (European Parlament and Council, 2006/962/EC; UNESCO, 1972, 2014). Al mismo tiempo, se subraya "el valor y el potencial del patrimonio cultural utilizado prudentemente como recurso para el desarrollo sostenible y la calidad de vida de una sociedad en continua evolución" (Council of Europe, 2005, p. 2). Por tanto, el patrimonio cultural se convierte en elemento fundamental en el desarrollo de ciudadanos de pleno derecho con una identidad cultural que va desde un ámbito local, hasta el desarrollo de una identidad nacional, europea y global (Copeland, 2006).

España cuenta con un gran volumen de patrimonio que es necesario gestionar. A este respecto la educación patrimonial se convierte en una disciplina de pleno derecho, porque solo mediante la apropiación de la sociedad de los valores culturales presentes en el patrimonio podemos vislumbrar un camino de sostenibilidad en la salvaguarda de los mismos (Lobovikov-Katz, 2009). La educación patrimonial se ha consolidado en nuestro país como una disciplina científica con un amplio desarrollo académico. Reflejo de ello son el gran número de proyectos de I+$\mathrm{D}+\mathrm{i}$, tesis doctorales y publicaciones en revistas de alto impacto en torno a la misma (Fontal e Ibáñez-Etxeberria, 2017).

A nivel nacional, también ha sido constante la preocupación en materia de patrimonio y educación por parte del Estado, que encuentra su punto álgido en el año 2013 cuando se implanta el Plan Nacional de Educación y Patrimonio (Domingo, Fontal y Ballesteros, 2013), que se configura como un instrumento que permite coordinar las administraciones autonómicas y estatales en materia de educación y patrimonio. Sin duda, este marco normativo sienta las bases para articular actuaciones de sensibilización destinadas a la sociedad en su totalidad y al conjunto de ámbitos educativos y contextos sociales. Al mismo tiempo supone una hoja de ruta sobra la cual las comunidades autónomas deben diseñar acciones más específicas, concretas, sistematizadas y adecuadas a las necesidades sociales de sus entornos patrimoniales y de su población.

La Comunidad de Madrid, desde la Dirección General de Patrimonio Cultural ha recogido el testigo de la Subdirección General del Instituto de Patrimonio Cultural de España e incorpora el concepto de educación patrimonial para el desarrollo de sus principales competencias que son la conservación, sensibilización, promoción y difusión del patrimonio cultural de la comunidad. Así como, la promoción del enriquecimiento del mismo, y el fomento y tutela del acceso de los ciudadanos a los bienes comprendidos en él. Para alcanzar dichos fines se desarrollan comisiones que están trabajando en el desarrollo de planes de actuación, entre los que se encuentra el Plan de Educación Patrimonial que recoge objetivos y líneas maestras de actuación. Este plan conecta los ámbitos formal, no formal e informal de la educación (Fontal, Ibáñez-Etxeberria, Martínez y Rivero, 2017), fomenta la colaboración entre administraciones privadas y públicas, y desarrolla acciones y productos educativos que cubran las necesidades particulares de los diversos públicos, poniendo de manifiesto la necesidad de que diferentes profesionales de una misma materia trabajen conjuntamente en la elaboración de propuestas didácticas que interactúen entre los distintos medios (Berbel y Díaz, 2014). Con la intención de conocer los diversos públicos a los que irán destinadas las acciones y productos edu- cativos se desarrolla la presente investigación, que tiene como objetivo principal describir y analizar la percepción que tienen las personas que acudieron a la XI Bienal Ibérica del Patrimonio Cultural AR\&PA del patrimonio de la Comunidad de Madrid, a través del diseño y aplicación práctica de una entrevista semiestructurada. Para cumplir dicho objetivo, la entrevista plantea cuestiones acerca de variables referidas a la percepción del patrimonio como la tipología patrimonial que recuerda el entrevistado que haya visitado la Comunidad de Madrid, así como su opinión acerca de la accesibilidad a dicho patrimonio y su relación con el mismo, pudiendo ser profesional, académica, de ocio, etc. Además, los resultados obtenidos son correlacionados con los resultados de la encuesta propuesta por Cepeda y Fontal (2019) acerca de la tipología patrimonial a la que recurren las personas cuando han de pensar en el patrimonio de un lugar, con el fin de averiguar si existe una tipología predominante en ambos casos.

\section{La Educación patrimonial y la significación social del Patri- monio Cultural}

La Carta de Atenas de 1931 argumentaba que cualquier política de protección y fomento sobre un bien patrimonial que no goce del aprecio de la sociedad, estará condenada al fracaso a largo plazo (UNESCO, 1931, art. X). Este punto ya planteaba la importancia que tiene la sociedad en la gestión y conservación del patrimonio cultural, otorgando a las personas la capacidad de atribuir valores a los bienes culturales para convertirlos en patrimonio. Estos valores atribuidos han ido cambiando a lo largo de la historia con motivo del dinamismo inserto en la conceptualización del Patrimonio Cultural, como construcción social, cultural y política (Pocock, Collett, y Baulch, 2015). Así, nuevos valores identitarios han sido atribuidos a otro tipo de bienes culturales centrados en las expresiones y manifestaciones de una comunidad, incorporando la dimensión intangible al concepto de patrimonio cultural (Fontal y Martínez, 2017), que permite facilitar la evocación de los procesos históricos en los que se han gestado los bienes y comprender la significación social de los mismos.

La relación de la sociedad con el patrimonio y la cultura también ha ido cambiando a lo largo de la historia. En la segunda mitad del siglo XX se comienzan a desarrollar las políticas de democratización cultural, más concretamente a principios de los años 70, según Ariño (2010), cuando los gobiernos de los distintos países, bajo la creencia del valor civilizador de la cultura, trataron de democratizar el acceso a la misma (Matarasso y Landry, 1999). Los organismos internacionales como el Consejo de Europa y la UNESCO también han defendido la democratización cultural. En la Declaración de Bremen de 1983, el Consejo de Europa reivindicaba que las autoridades locales son las responsables de que "cada ciudadano tome parte en la vida cultural de la ciudad" (Council of Europe, 1983, art. 14). También la UNESCO, ya en su Declaración de México de 1982, declaraba que “Una política cultural democrática hará posible el disfrute de la excelencia artística en todas las comunidades y entre toda la población (Unesco, 1982, art. 2).

Posteriormente, en el año 2005 la Convención Marco del Consejo de Europa sobre el Valor del Patrimonio Cultural para la Sociedad se plantea el derecho de todos los ciudadanos a participar en la vida cultural, ya enunciado en la Declaración Universal de los Derechos Humanos (ONU, 1948). La Convención presenta la educación como herramienta de diálogo entre culturas y el patrimonio cultural como recurso para revindicar la diversidad como factor potencial de la creatividad humana, que mejora en entendimiento social y la convivencia pacífica (Council of Europe, 2005, art. 1). Aunque ya la Recommendation No. $R$ (98) 5 concerning heri- 
tage education, que supuso el comienzo en la construcción de un marco formalizando en materia de educación y patrimonio, que recomienda a los Estados miembros adoptar las medidas legislativas, reglamentarias, administrativas, financieras y de otro tipo para iniciar y desarrollar programas educativos sobre patrimonio, con la finalidad de promover la conciencia del patrimonio entre los jóvenes (Committee of Ministers, 1998). Todo ello se plantea con la necesidad de cultivar una relación sostenible, desde una perspectiva más ética y educativa, entre la población y el patrimonio cultural, donde la población se implique en los procesos culturales, convirtiéndose en protagonista de los mismos. En este sentido, la educación patrimonial cobra un papel fundamental en tanto tiene como principal objetivo sensibilizar a la sociedad. Una vez que la población ha sido sensibilizada con determinada realidad, según Fontal (2003), muestra una disposición a cuidarla, disfrutarla y transmitirla, como consecuencias directas. Este empoderamiento de la población a través de la educación supone el motor de cambio hacia la gestión sostenible del patrimonio.

El punto de partida para el desarrollo de cualquier acción educativa, tanto en el ámbito formal como no formal (Berbel y Díaz, 2014), debe ser la evaluación de los sujetos para los que se va a diseñar dicha acción. Por ello, las administraciones públicas encargadas de la gestión patrimonial necesitan diseñar instrumentos de evaluación para conocer los conocimientos previos, intereses, inquietudes y el grado de sensibilización hacia el patrimonio de los destinatarios, en base al cual realizar el diseño educativo. La evaluación es considerada como una de las actividades de responsabilidad preferente, ya que no hay actuación tecnificada ni sistémica sin evaluación (Sarramona y Rodríguez, 2010). La evaluación debe entenderse en sentido amplio, abarcando todos los elementos que la integran: "procesos y productos, pero también recursos, estructuras" (Sarramona y Rodríguez, 2010, p. 9), donde la evaluación de los conocimientos previos debe ser el punto de partida.

Existen investigaciones que tienen como principal finalidad conocer la significación social del patrimonio para un determinado grupo social (Cepeda y Fontal, 2019; Llull, 2005; Morate, 2007; Matthews et al., 2019). En este sentido también encontramos conexiones con investigaciones que argumentan la necesidad de evaluar los resultados de esos programas educativos. La investigación desarrollada por Ortiz et al. (2018) desarrolla una encuesta sobre la opinión de los visitantes para estudiar el impacto de un nuevo modelo educativo que busca difundir la investigación en preservación del patrimonio cultural, y evaluar los resultados de la experiencia. E investigaciones que plantean la importancia de evaluar también el diseño educativo de los propios programas (Rivero, Fontal, Martínez y García-Ceballos, 2018). A pesar de las investigaciones desarrolladas sigue existiendo la necesidad de ahondar en las principales ideas y preocupaciones que la mayoría de los ciudadanos tienen en torno al patrimonio cultural, para sensibilizar a la población y lograr su empoderamiento (Texeira, 2006).

\section{Método}

El estudio trata de analizar un concepto cualitativo como es la percepción social del patrimonio de la Comunidad de Madrid con el fin de ofrecer una visión cuantitativa que nos aporte las claves para enfocar futuros programas que refuerce los puntos débiles observados tras el análisis.

Para ello, se ha elaborado una entrevista semiestructurada (ver Anexo II) dividida en dos apartados: el primero relativo a los datos sociodemográficos de los entrevistados y el segundo referido a variables relativas a la percepción sobre el patrimonio de la Comunidad de Madrid.

La recogida de información tuvo lugar en un único momento temporal comprendido entre el 8 y 11 de noviembre de 2018 en el Centro Cultural Miguel Delibes de Valladolid, durante la celebración de la XI Bienal Ibérica del Patrimonio Cultural AR\&PA. La muestra resultante fue un total de $n=122$ individuos. Las entrevistas fueron guiadas por un investigador experto en el tema y los entrevistados participaron de manera voluntaria.

Con respecto a la protección de datos, todos los participantes firmaron siempre un consentimiento (ver Anexo I), ya que todas las entrevistas fueron grabadas con el fin de elaborar un audiovisual para la propia Dirección General de Patrimonio Cultural de la Comunidad de Madrid. Para llevar a cabo el análisis estadístico-descriptivo se ha utilizado el software Statistical Product and Service Solutions (IBM Analytics), versión 21.

\subsection{Procedimiento}

Para el diseño de la entrevista, se ha partido del diseño del cuestionario elaborado por Cepeda y Fontal (2019) con el que trataban de medir el grado de vinculación identitaria entre un individuo y el patrimonio de su entorno concreto. Para ello, se ha elaborado una entrevista con dos secciones diferenciadas:

$1^{\circ}$ Apartado. Variables sociodemográficas.

- Edad.

- Género.

- Localidad de procedencia. Se trata de una respuesta abierta en la que el individuo declara su lugar de residencia.

- Nivel de estudios. Esta variable adquiere tres valores: estudios primarios, secundarios y superiores.

$2^{\circ}$ Apartado. Variables relativas a la percepción sobre el patrimonio de la Comunidad de Madrid.

- Motivación de la presencia en la Bienal de AR\&PA. Esta variable puede adquirir seis valores diferentes: Motivación académica, profesional, familiar, política, personal, trabajador en AR\&PA e interés cultural.

- Tipología patrimonial de la Comunidad de Madrid. Se trata de una respuesta abierta en la que el entrevistador podía ayudar a identificar la tipología patrimonial a raíz del bien patrimonial ofrecido por el entrevistado. Esta variable puede tomar hasta diez valores diferentes: Arqueológico, arquitectónico, artístico, cultural, inmaterial, histórico, monumental, natural, pictórico y variado.

- Secuencia de sensibilización. Esta variable se refiere al disfrute, al sentimiento de identidad y a la difusión del patrimonio y puede tomar como valores la secuencia completa o de manera parcial.

- Accesibilidad al patrimonio. A través de una pregunta dicotómica en la que el entrevistado debe responder si piensa que todas las personas independientemente de su estado físico y/o psíquico pueden acceder al patrimonio de la Comunidad de Madrid.

- Conocimiento sobre el Plan Nacional de Educación y Patrimonio. Se trata de otra pregunta dicotómica que tiene por fin averiguar si las personas conocen por sí mismas o a través de terceros el Plan Nacional de Educación y Patrimonio.

\subsection{Validación}

La entrevista fue validada por 8 miembros del Observatorio de Educación Patrimonial en España, pertenecientes al equipo 
de investigación del proyecto (Ref. EDU 2015/65716-C2-1-R) y 2 expertos pertenecientes a la Dirección General de Patrimonio Cultural de la Comunidad de Madrid, que actuaron de "árbitros de control de la fiabilidad del análisis, que ayudaron a perfilar y enriquecer las distintas categorías" (López-Noguero, 2002, p. 176). Así mismo, la entrevista fue sometida a pruebas piloto con una muestra aleatoria de tres individuos de distintas edades para verificar la comprensión de las preguntas.

\section{Resultados}

Los resultados que describen el comportamiento del total de la muestra $n=122$ individuos se presentan en diferentes tablas y gráficos, en los que se expresan los porcentajes de respuesta de los individuos que han contestado a cada una de las preguntas. En la tabla 1 se recoge la información relativa a las características sociodemográficas de la muestra. Se puede comprobar que la mayor parte de las personas que han respondido son mujeres $(63,11 \%)$, residentes en alguna localidad de la comunidad autónoma de Castilla y León $(82 \%)$, más concretamente en Valladolid $(47,5 \%)$, con una media de edad de alrededor de 40 años. El nivel de estudios de los encuestados es principalmente superior $(77,87 \%)$.

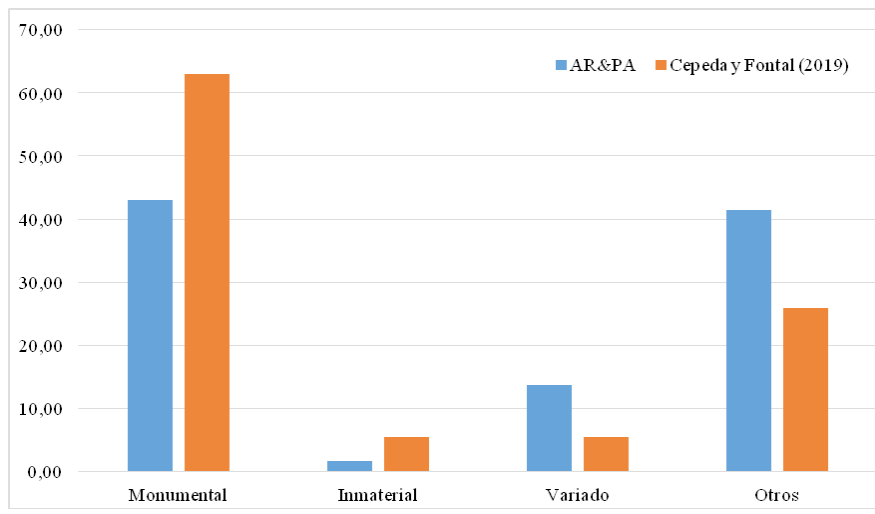

Gráfico 1. Comparación tipología patrimonial.

Una vez descritas las características sociodemográficas de la muestra, continuamos con la exposición de los resultados obtenidos a través de la segunda fase de la entrevista relativa a la relación entre el patrimonio y las personas, así como la motivación por la que los encuestados acudían a un evento como es la Feria de AR\&PA. Además, se exponen los datos obtenidos relativos a las opiniones sobre la accesibilidad al patrimonio de las personas con algún tipo de diversidad funcional y el conocimiento o desconocimiento de la existencia de algún plan o documento normativo, que regule aspectos sobre educación y patrimonio en la Comunidad de Madrid.

En la tabla 2 se muestra la motivación de las personas entrevistadas en asistir a la XI Bienal Ibérica de Arte y Patrimonio, donde se puede comprobar que los principales motivos son profesionales y académicos, sumando casi el 50\% del total entre ambos. Muy seguidos de ellos se encuentran los motivos personales (20,5\%) y ser partícipes de AR\&PA como trabajadores o voluntarios $(\mathbf{1 6 , 4 \% )}$.

En la tabla 3 se detallan las diferentes tipologías patrimoniales a las que aludieron los entrevistados por su propia iniciativa, sin partir de categorías planteadas previamente. El entrevistador, en el momento que detectaba alguna dificultad en el entrevistado para identificar dicha tipología a la que deseaba referirse, ayudaba mínimamente convirtiendo los ejemplos patrimoniales que ex-

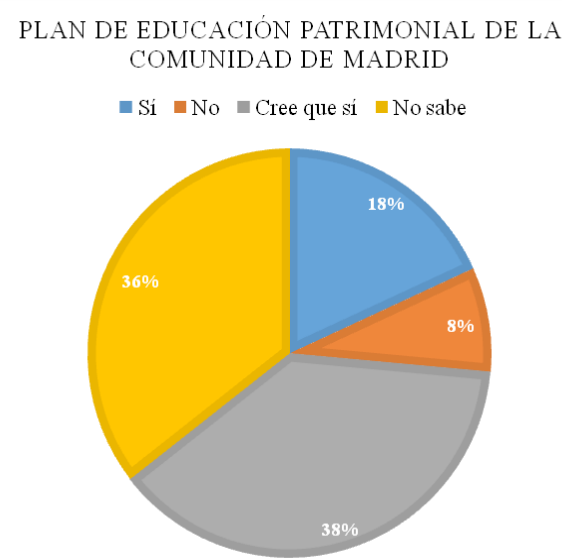

Gráfico 2. Plan de Educación Patrimonial de la Comunidad de Madrid.

ponían en la tipología que correspondiese en cada caso, tomando como referencia las planteadas por UNESCO en sus convenciones (2005; 2014). De este modo, las respuestas de los entrevistados se fueron codificando al instante con el fin de homogeneizar los resultados y poder realizar un análisis estadístico de las categorías en las que se encuadraban los elementos patrimoniales citados. A modo de ejemplo, fueron nombrados bienes patrimoniales tales como el Museo del Prado (cultural y monumental), el casco viejo de la ciudad de Madrid (arquitectónico), la Sierra de Guadarrama (natural), el Palacio Real (monumental) así como la variedad de musicales y obras de teatro a las que poder acudir (inmaterial). No obstante, como se puede ver en la tabla, la mayor parte de las personas recordaban algún bien patrimonial monumental $(45,5 \%)$ de la Comunidad de Madrid o cultural (22,3\%), relegando al resto de tipologías a un segundo plano.

Tabla 1

Información sociodemográfica de la muestra

\begin{tabular}{|c|c|}
\hline Variable & Porcentaje de respuesta \\
\hline \multicolumn{2}{|l|}{ Sexo } \\
\hline Hombre & $36,89 \%$ \\
\hline Mujer & $63,11 \%$ \\
\hline \multicolumn{2}{|l|}{ Lugar de procedencia } \\
\hline ESPAÑA & $97,6 \%$ \\
\hline Aragón & $0,8 \%$ \\
\hline Castilla y León & $82 \%$ \\
\hline Cantabria & $1,6 \%$ \\
\hline Comunidad de Madrid & $9 \%$ \\
\hline Comunidad Valenciana & $1,6 \%$ \\
\hline Extremadura & $1,6 \%$ \\
\hline País Vasco & $0,8 \%$ \\
\hline PORTUGAL & $1,6 \%$ \\
\hline ESCOCIA & $0,8 \%$ \\
\hline Edad & $39,67^{*}$ \\
\hline \multicolumn{2}{|l|}{ Nivel de estudios } \\
\hline Primarios & $1,64 \%$ \\
\hline Secundarios & $20,49 \%$ \\
\hline Superiores & $77,87 \%$ \\
\hline
\end{tabular}

*Media de la variable expresada en años 
Como se ha comentado a la hora de describir de manera sociodemográfica la muestra, la mayor parte de los entrevistados procedían de Valladolid, por lo que se consideró pertinente comparar las respuestas de los vallisoletanos respecto a la tipología patrimonial con la encuesta de Cepeda y Fontal (2019).. Para ello, se recopilaron las 58 respuestas de las personas que residen en Valladolid de la pregunta 7 (ver Anexo II) y las 54 de la pregunta siguiente: 3. ¿Conoce algún bien patrimonial que sea representativo de la localidad en la que reside? 3b. ¿Qué tipo de patrimonio es? Como resultado, a partir de la figura 1, se observa cómo en ambas encuestas las personas recurren normalmente al patrimonio monumental dejando al margen el resto de tipologías planteadas por UNESCO. En la variable "Otros", se han agrupado en el caso de la entrevista de AR\&PA tipologías como arquitectónico, arqueológico, cultural, histórico, natural y pictórico, y en el de la encuesta sobre la identidad cultural de Castilla y León, etnológico, caminería histórica, industrial y urbanístico, con el fin de poder realizar una comparación gráfica. De esta manera, se puede indicar que, aun agrupando todas estas categorías en una sola variable, la tipología monumental sigue por encima en ambas encuestas.

Tabla 2

Motivación

\begin{tabular}{lc}
\hline & Porcentaje \\
\hline Académica & 23,8 \\
Profesional & 25,4 \\
Trabajador en AR\&PA & 16,4 \\
Familia & 10,7 \\
Interés cultural & 2,5 \\
Política &, 8 \\
Personal & 20,5 \\
\hline
\end{tabular}

Una vez que los entrevistados expusieron el tipo de patrimonio que recordaban de la Comunidad de Madrid, se les preguntó qué les unía a dicho patrimonio obteniendo respuestas muy dispares que giraban en torno a intereses profesionales y académicos, ya que varias personas se encontraban realizando estudios relacionados con el arte y la música o se dedicaban a la gestión cultural, al ocio, al gusto o placer, habiendo mayoría de individuos que visitaban la Comunidad de Madrid únicamente por turismo, y a la familia, puesto que varias personas tenían algún familiar músico, pintor o actor.

Tabla 3

Tipología patrimonial

\begin{tabular}{lc}
\hline & Porcentaje \\
\hline Arqueológico & 2,5 \\
Arquitectónico & 5,8 \\
Artístico & 4,1 \\
Cultural & 22,3 \\
Inmaterial & 7,4 \\
Histórico & 1,7 \\
Monumental & 45,5
\end{tabular}

\begin{tabular}{lc}
\hline & Porcentaje \\
\hline Natural & 2,5 \\
Pictórico & 1,7 \\
Variado & 6,6 \\
\hline
\end{tabular}

En la tabla 5 se abordan las inercias positivas derivadas de la secuencia de sensibilización planteada por Fontal (2003), que fue simplificada en 3 ítems con el fin de facilitar el entendimiento al entrevistado. De este modo, dichos ítems hacen referencia al disfrute de las personas entrevistadas con el patrimonio de la Comunidad de Madrid, a la difusión de dicho patrimonio a través de las redes sociales principalmente y al sentimiento de identidad con dicho patrimonio, independientemente de la procedencia del entrevistado. Se puede comprobar que más del $80 \%$ de los entrevistados responde positivamente a esta secuencia (disfruta - comparte - se identifica), sintiendo el patrimonio de esta comunidad como parte de su propia identidad.

Tabla 4

Secuencia de sensibilización

\begin{tabular}{lc}
\hline & Porcentaje \\
\hline Disfruta/Difunde/Identifica & 84,3 \\
Disfruta/Difunde & 9,1 \\
Disfruta/Identifica & 3,3 \\
Disfruta & 1,7 \\
Difunde &, 8 \\
Difunde/Identifica &, 8 \\
\hline
\end{tabular}

Por otro lado, se consideró pertinente preguntar acerca de la accesibilidad al patrimonio, con el objetivo de recoger las percepciones generales de las personas que han visitado cualquier bien patrimonial de la Comunidad de Madrid. Tras analizar la tabla 6 , se observa que existen dos respuestas mayoritarias totalmente opuestas en este aspecto, puesto que el $41 \%$ de los entrevistados opinan que sí hay total accesibilidad al patrimonio para todos, mientras que el 40,2\% piensan todo lo contrario. Además, el 18\% cree que se debería mejorar en este aspecto.

Tabla 5

Accesibilidad al patrimonio

\begin{tabular}{lc}
\hline & Porcentaje \\
\hline Sí & 41,0 \\
No & 40,2 \\
Debería mejorar & 18,0 \\
No sabe &, 8 \\
Total & 100,0 \\
\hline
\end{tabular}

Para finalizar, se plantea una última cuestión con el fin de comprobar el conocimiento de las personas entrevistadas acerca de la existencia de algún plan o documento normativo que regule todos los temas relacionados con la educación y el patrimonio en la Comunidad de Madrid, se puede ver, a través de la figura 
3, que hay un cierto desconocimiento, puesto que el 36\% no sabe de su existencia, el $8 \%$ piensa que no existe y sólo un $18 \%$ sabe con certeza de su existencia.

\section{Conclusiones}

Tras la exposición de todos los resultados, se puede constatar que la Comunidad de Madrid es un punto clave, tanto como medio de paso como destino final para numerosos turistas, ya que todos los entrevistados afirmaron que habían estado de visita en la ciudad de Madrid y/o en algún municipio de la comunidad. Este hecho reafirma la necesidad de la colaboración entre entidades públicas y privadas para garantizar una gestión adecuada del patrimonio cultural de la Comunidad de Madrid con el fin de afianzar el sentimiento de disfrute entre los visitantes con el patrimonio, un patrimonio que actuará como elemento de conexión social (Teixeira, 2006).

Si hacemos hincapié en los resultados más relevantes de la entrevista, se observa que, de las 122 personas entrevistadas, 115 difunden el patrimonio de la Comunidad de Madrid, aunque no lo hacen de la misma manera. La mayor parte de las personas que se encuentran en AR\&PA por una motivación profesional presentan una relación directa con el patrimonio, ya que su trabajo gira entorno a él. Por ello, los gestores culturales y las personas encargadas de la promoción en empresas culturales realizan una difusión masiva vía redes sociales y web de manera muy regular. Este tipo de difusión podemos considerarla de tipo profesional, aunque todas las personas entrevistadas que trabajan para y por el patrimonio contestaron que disfrutan con él, por lo que también realizan una difusión del patrimonio visitado a través de sus perfiles personales en las redes sociales. Por otra parte, los jóvenes que han nacido ya en una era totalmente tecnológica ofrecen respuestas muy esclarecedoras que confirman este hecho, ya que prácticamente todos comparten fotos y vídeos de sus recuerdos patrimoniales por redes sociales como Facebook, Instagram o Twitter. Gracias a los nuevos sistemas de geolocalización, basta con realizar una foto con nuestro Smartphone para que este mismo te indique el monumento que encuentras fotografiando. Además, también ha sido posible comprobar cómo aún hay personas, aunque no demasiadas y todas de edad adulta o avanzada, que difunden su contacto con el patrimonio de Madrid a través del "boca a boca" o por medio de la fotografía en formato físico, ya sea con su familia o sus amigos. Por lo tanto, se observan diferentes patrones de conducta según la edad y la profesión.

Además, siguiendo con la misma cuestión acerca de la secuencia de sensibilización propuesta por Fontal (2003, pág. 209): conocer-comprender-valorizar-sensibilizar-preservar-disfrutar-transmitir-sociabilizar-identizar, y que fue simplificada en la entrevista en únicamente tres acciones (disfrutar-difundir-identificar), cabe destacar positivamente que se cumple en un porcentaje muy elevado, lo cual quiere decir que la población muestra inercias positivas hacia el patrimonio, denotando cierta sensibilización hacia el mismo. No obstante, se debe indicar que la mayor parte de las personas que han contestado a la entrevista se relacionan con el patrimonio por motivos académicos, profesionales o de ocio, por lo que es difícil generalizar este hecho a toda la población que haya visitado la Comunidad de Madrid.

Otro aspecto que suscitó dudas a los entrevistados fue el tema de la accesibilidad al patrimonio. Mientras una gran parte opinaban que no existe una accesibilidad total al patrimonio y que queda mucho que trabajar en este aspecto, otros consideraban que sí se ha trabajado y se sigue trabajando en este camino. Las personas más optimistas sobre este tema, respaldaban su opinión en las grandes adaptaciones que han comprobado presentan los museos de la comunidad de Madrid. No obstante, la mayor parte de los ejemplos que recordaban, hacían alusión únicamente a las modificaciones realizadas para personas con movilidad reducida. Independientemente de la discrepancia de opiniones a este respecto, todos ellos coincidían en la necesidad de seguir trabajando en este camino, ya que por ley todo el mundo debería tener acceso al patrimonio (Fontal y Marín, 2018).

Por otro lado, ha quedado constancia de que las personas recurren normalmente al patrimonio monumental cuando han de pensar en su experiencia con el patrimonio en general, tanto de la Comunidad de Madrid como de Castilla y León, dejando de lado el patrimonio inmaterial. Se sigue percibiendo el patrimonio como algo tangible, lo cual hace olvidarnos de la fuerte proyección teatral, musical y cinematográfica de Madrid, así como de las tradiciones y fiestas populares que congregan a miles de personas en pueblos de Castilla y León multiplicando su población por dos, tres o cuatro durante varios días, etc. El hecho de relegar el patrimonio inmaterial a un segundo plano, demostrado a raíz de las respuestas ofrecidas en ambas encuestas, debe ser el punto de partida para poder establecer acciones educativas y de sensibilización patrimonial que se ajusten a las necesidades sociales y no fomenten el olvido de este tipo de patrimonio. Ese punto de partida debería concretarse con la puesta en marcha del Plan de Educación Patrimonial de la Comunidad de Madrid, pudiendo ser de ayuda para asociaciones públicas o privadas, centros educativos, museos y cualquier sector o disciplina relacionada con el patrimonio, facilitando la comunicación entre todos los profesionales del sector.

Este análisis pretende ser el punto de partida de futuras investigaciones, ya que conocer el grado de sensibilización de la población hacia el patrimonio nos permitirá realizar acciones educativas enfocadas a las necesidades sociales que tengan como principal finalidad empoderar a la población en la gestión sostenible de sus bienes patrimoniales (Lobovikov-Katz, 2009).

\section{Referencias bibliográficas}

Diseño de una encuesta para indentificar las formas de relación identitaria en Castilla y León. Revista Electrónica Interuniversitaria de Formación del Profesorado, 22 (1), 29-40.

Berbel, N., y Díaz, M. (2014). Educación formal y no formal. Un punto de encuentro en educación musical. Aula abierta, 42(1), 47-52.

Copeland, T. (2006). European democratic citizenship, heritage education and identity. Strasbourg, France: Council of Europe.

Council of Europe. (1983). Bremen Declaration. New response for cultural problems Council of Europe Framework Convention on the Value of Cultural Heritage for Society (CPL/ CCC (83) 11-E). Retrieved from https://rm.coe.int/CoERMPublicCommonSearchServices/DisplayDCTMContent?documentId=09000016806b23f4.

Council of Europe. (2005). Council of Europe Framework Convention on the Value of Cultural Heritage for Society (Faro, 27.X.2005). Council of Europe Treaty Series, 199. Retrieved from http:/ / conventions.coe.int/Treaty/EN/Treaties/Html/199. htm.

Cepeda, J., y Fontal, O. (2019). Diseño de una encuesta para identificar las formas de relación identitaria en Castilla y León. Revista Electrónica Interuniversitaria de Formación del Profesorado, 22(1), 29-40.

Domingo, M., Fontal, O., y Ballesteros, P. (Coords.). (2013). Plan Nacional de Educación y Patrimonio. Madrid: Ministerio de Educación, Cultura y Deporte, Secretaría de Estado de Cultura. 
Fontal, O. (2003). La Educación Patrimonial. Teoría y práctica en el aula, el museo e internet. Gijón: Trea.

Fontal, O., e Ibáñez-Etxeberria, A. (2017). La investigación en Educación Patrimonial. Evolución y estado1 actual a través del análisis de indicadores de alto impacto. Revista de Educación, 375(1), 84-214.

Fontal, O., Ibáñez, A., Martínez, M. y Rivero, P. (2017). Heritage as content in Primary Education: From the Curriculum to Teacher Education. Revista Electrónica Interuniversitaria de Formación del Profesorado, 20(2), 79-95. http:/ /dx.doi.org/10.6018/reifop.20.1.286321.

Fontal, O., y Martínez M. (2017). Evaluación de programas educativos sobre Patrimonio Cultural Inmaterial. Estudios Pedagógicos, 43(4), 69-89. http:/ /dx.doi.org/10.4067/S071807052017000400004

Fontal, O., y Marín, S. (2018). Inclusive heritage: towards an index for the evaluation of educational inclusion to heritage. CADMO, 44, 117-120.

Llull, J. (2005). Evolución del concepto y de la significación social del patrimonio cultural. Arte, Individuo y Sociedad, 17, 175-204.

Lobovikov-Katz, A. (2009). Heritage Education for Heritage Conservation. A Teaching Approach, Strain, 45(1), 480-484. https:// doi.org/10.1111/j.1475-1305.2008.00569.x

López-Noguero, F. (2002). El análisis de contenido como método de investigación. XXI Revista de Educación, 4(1), 167-179.

Matarasso, F. y Landry, C. (1999). Balancing act: twenty-one strategic dilemmas in cultural policy. Strasbourg: Council of Europe.

Matthews, R., Hussain, O., Palmero, M., Fobbe, S., Nováček, K., Mohammed-Amin, R... Richardson, A. (2019). Heritage and cultural healing: Iraq in a post-Daesh era. International Journal of Heritage Studies. https:// doi.org/10.1080/13527258.2019.1 $\underline{608585}$
Morate, G. (2007). Conocimiento y percepción del patrimonio histórico en la sociedad española. Revista electrónica de patrimonio histórico, 1, 1-10.

Ortiz, P., et al. (2018). The Hidden Face of Cultural Heritage: a science window for the dissemination of elementary knowledge of risk and vulnerability in cultural heritage. Heritage Science, 60, 2-15. https://doi.org/10.1186/s40494-018-0224-z

Rivero, P., Fontal, O., Martínez, M., y García-Ceballos, S. (2018). La educación patrimonial y el patrimonio arqueológico para la enseñanza de la Historia: el caso de Bílbilis. Ensayos. Revista de la Facultad de Educación de Albacete, 33(1), 23-37. http:// www.revista.uclm.es/index.php/ensayos

Sarramona, J., y Rodríguez, T. (2010). Participación y calidad de la educación. Aula abierta, 38(1), 3-14.

Teixeira, S. (2006). Educación patrimonial: alfabetización cultural para la ciudadanía. Estudios Pedagógicos, 32, 133-145.

UNESCO. (1972). Convention for the Protection of the World Cultural and Natural Heritage. Paris: UNESCO. Recuperado de https://whc.unesco.org/document/163850

UNESCO. (2005). Convention on the Protection and Promotion of the Diversity of Cultural Expressions. Paris: UNESCO. Recuperado de http://unesdoc.unesco.org/images/0014/001429/142919e.pdf

UNESCO. (2014). Managing Cultural World Heritage. Paris: UNESCO. Recuperado de https://whc.unesco.org/en/managing-cultural-world-heritage/ 
Anexos

Autorización

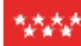

Direccion General de Patrimonio curtural CONSEJERIA DE CULTURA.

Comprnided

D./Dña.

Autorizo la fijación en soporte audiovisual de mi participación, como sujeto entrevistado en el stand de la Comunidad de Madrid, en la feria AR\&PA 2018 XI Bienal Ibérica del Patrimonio Cultural, realizada el día de noviembre de 2018 en el Centro Cultural Miguel Delibes en Valladolid, dentro del proyecto del Plan de Educación Patrimonial de dicha Comunidad. Y por la presente, realizo la cesión no exclusiva de los derechos de reproducción y comunicación pública de dicha fijación, en todo el mundo y sin límite de tiempo, a los efectos de su publicación y posterior difusión en la página web y canales de redes sociales de la Comunidad de Madrid.

En Valladolid, a de del 2018

Fdo. :

DNI:

\section{Entrevista}

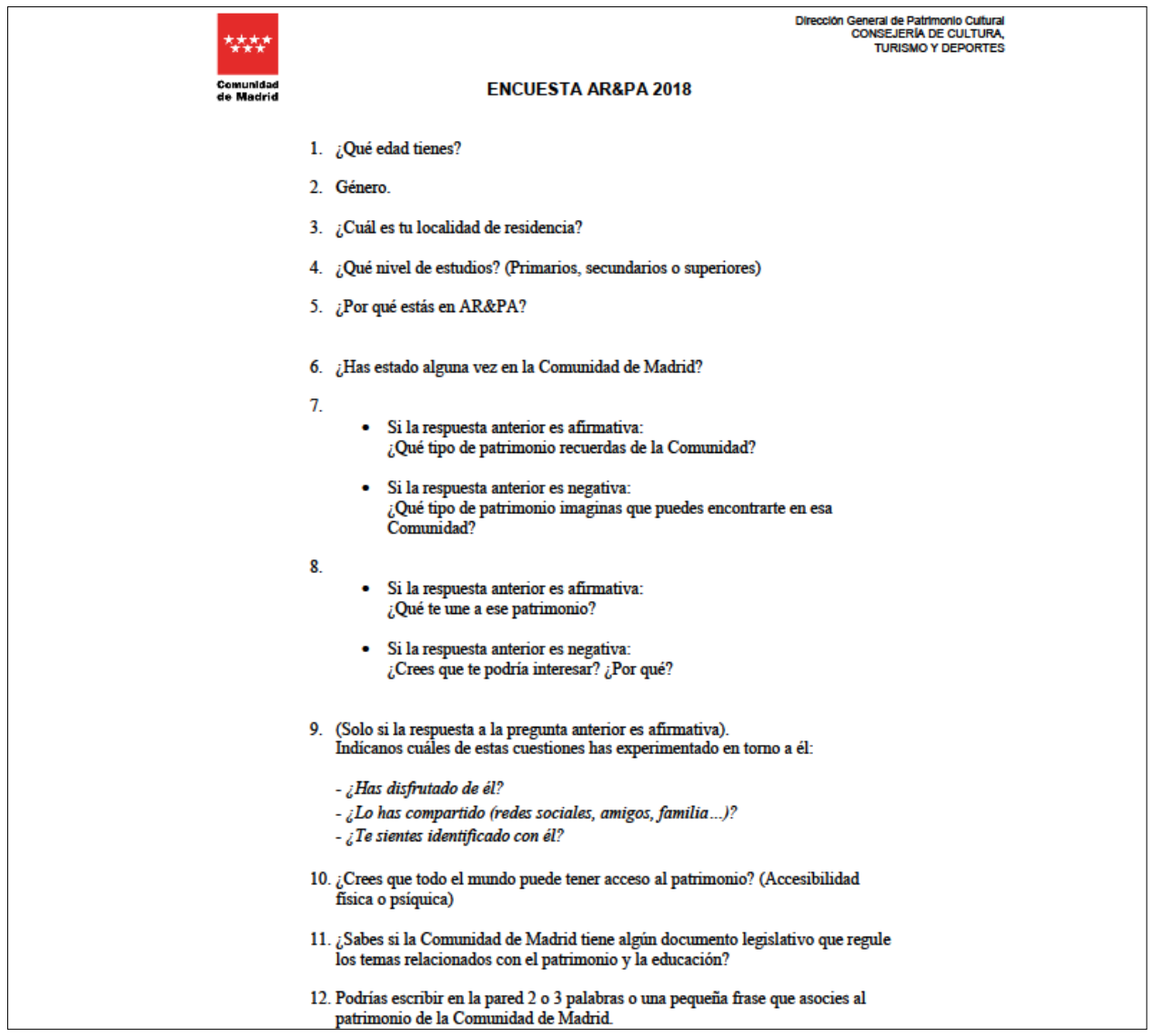

